\title{
E-Mode Propagation in a Plane-Stratified Plasma ${ }^{1}$
}

\section{Peter Hirsch}

\author{
Bell Telephone Laboratories, Inc., Whippany, N.J. \\ and \\ Jerry Shmoys \\ Department of Electrophysics, Polytechnic Institute of Brooklyn
}

(Received October 16, 1964; revised November 27, 1964)

\begin{abstract}
The problem of propagation of plane electromagnetic waves polarized in the plane of incidence in a plane-stratified isotropic plasma (regarded as a dielectric) is dealt with by reducing it to the solution of an ordinary differential equation which is singular at the level of plasma resonance. The absorption of power in the resonant layer is calculated approximately in two cases involving a linear profile. In the first the relative dielectric constant varies linearly in a thin transition layer between two homogeneous regions. In the second, the dielectric constant is linearly varying over a large interval.
\end{abstract}

\section{Introduction}

The problem of the reflection of a plane electromagnetic wave from a plane stratified plasma presents considerably more difficulty in the "vertical" polarization case (i.e., in the case of electric field in the plane of incidence) than in the "horizontally" polarized case [Budden, 1961]. In the latter, the ordinary differential equation which one obtains after eliminating the variables transverse to the stratification gradient exhibits a turning point at some level of the stratification, while in the former case, in addition to the turning point, the differential equation has a singularity. This singularity occurs at the stratum of plasma resonance.

We will assume that the plasma is an isotropic dielectric medium, with the dielectric constant related to the electron density $N(z)$ by the well-known formula

$$
\epsilon=\epsilon_{0}\left(1-N e^{2} / m \epsilon_{0} \omega^{2}\right)
$$

where $e$ and $m$ are the charge and mass of the electron, and with permeability equal to that of free space. We assume further that a time harmonic wave (with time dependence $\epsilon^{j \omega t}$ ) propagates in the medium. The physical inconsistencies arising from power loss at the level where $\epsilon=0$ in a lossless medium are resolved by appealing to the infinite duration of the transient process [Ginzburg, 1961], to nonlinearities which must occur when the fields become large [Budden, 1961], to coupling to plasma waves [Denisov, 1957], or to the impropriety of the dielectric model for the plasma, or they are avoided by considering a lossy plasma [Wait and Walters, 1963]. We will not concern ourselves here with these explanations. We shall merely obtain an approximate solution of the differential equation for a linearly varying electron density. The results will then be compared to those obtained previously by numerical methods [Budden, 1961].

Taken from the dissertation submitted by Peter Hirsch to the Faculty of the Brooklyn Polytechnic Institute in partial fulfillment of the requirements for the degree of Doctor of Philosophy, 1964. 


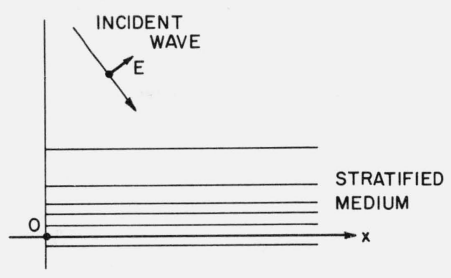

\section{Linear Profile}

In a plane stratified dielectric medium (see fig. 1), a plane wave incident in the $x z$-plane, polarized in the plane of incidence constitutes an $E$-mode with $H_{y}, E_{x}$, and $E_{z}$ as the only nonvanishing field components. After separating the field dependence on the $x$ coordinate through the factor $\exp \left(-j k_{x} x\right)$, we obtain the ordinary differential equation satisfied by $H_{y}$ :

$$
\frac{d^{2} H}{d z^{2}}-\frac{d \epsilon / d z}{\epsilon} \frac{d H}{d z}+\left(\omega^{2} \mu \epsilon-k_{x}^{2}\right) H=0
$$

where the subscript $y$ was omitted from the magnetic field $H$ since the other components are zero, and $\epsilon$ and $\mu$ denote the dielectric constant and permeability of the stratified medium. We see that the differential equation is singular at the zeros of $\epsilon$.

We shall consider in this paper two special cases, both characterized by a linearly varying dielectric constant. First we shall consider a transition from a region of constant negative dielectric constant to free space, assuming that the transition layer is thin. Second, we shall examine the relationship between the asymptotic forms of the solution of the problem of an infinite, linearly stratified medium on the two sides of the zero of the dielectric constant. Although the latter problem has no physical significance per se, it yields the connection formula necessary for the asymptotic solution of the problem of propagation in a medium whose stratification is both more complicated and more realistic.

If we introduce a linearly varying dielectric constant

$$
\frac{\epsilon(z)}{\epsilon_{0}}=-\frac{k_{0} z}{\delta}
$$

where $\epsilon_{0}$ is the dielectric constant of free space, $k_{0}$ the free space wave number and $\delta$ is the slope parameter, and then transform the independent variable

$$
x=\delta^{-1 / 3} k_{0} z,
$$

the differential equation (2) takes the form

$$
\frac{d^{2} H}{d x^{2}}-\frac{1}{x} \frac{d H}{d x}-(x+B) H=0
$$

where

$$
B=\delta^{2 / 3}\left(k_{x} / k_{0}\right)^{2}
$$

The differential equation is singular at the origin. When $B=0$, this singularity is only superficial. 
In this case the direction of incidence is parallel to the stratification gradient, and the solutions of the differential equation are derivatives of the Airy functions [Budden, 1961]. We can express the general solution of (5) in terms of power series [Försterling, 1949]

$$
H=\bar{\gamma}\left[\gamma v_{1}(x)+v_{2}(x)\right]
$$

where

$$
\begin{gathered}
v_{1}(x)=\sum_{0}^{\infty} \alpha_{n} x^{n+2} \\
\alpha_{0}=1, \quad \alpha_{1}=0, \quad \alpha_{2}=\frac{B}{8}, \quad \alpha_{n}=\frac{\alpha_{n-3}+B \alpha_{n-2}}{n(n+2)} \\
v_{2}(x)=\frac{B}{2} v_{1}(x) \ln x+\sum_{0}^{\infty} \beta_{n} x^{n} \\
\beta_{0}=1, \quad \beta_{1}=\beta_{2}=0, \quad \beta_{n}=\frac{B \beta_{n-2}+\beta_{n-3}-B(n-1) \alpha_{n-2}}{n(n-2)} .
\end{gathered}
$$

If we choose to normalize our solution in such a way that $H(0)=1$, as is convenient in this problem, we simply set $\bar{\gamma}=1$ and retain $\gamma$ as the arbitrary constant to be determined from the boundary conditions of the problem.

To make use of this solution in a physical problem we must resolve the question of the proper sheet of the logarithmic function; this can be done by considering the medium to have small losses, or by requiring the singularity to be a power sink rather than a power source.

\section{The Thin, Rapidly Varying Layer}

If the linearly stratified layer occurs as a transition between two homogeneous regions (cf. fig. 2) of relative dielectric constants 1 and $-\epsilon_{3}$, we can solve the reflection problem by making use of the power series (7) and (8) directly. In order that the series converge rapidly, we shall assume that the layer is thin; i.e., that the values of the dimensionless variable $x$ at both boundaries of the layer be small compared to unity in magnitude.

Recognizing that in region III the wave is exponentially decaying, we apply, at $x_{3}=\delta^{2 / 3} \epsilon_{3}$ the boundary condition

$$
d H / d x=-\left(\sqrt{\epsilon_{3} \delta^{2 / 3}+B}\right) H .
$$

FIGURE 2. The thin, rapidly varying layer.

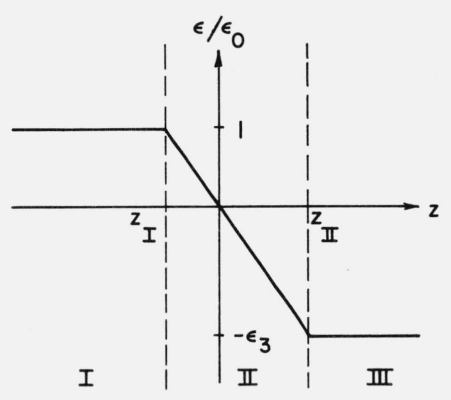




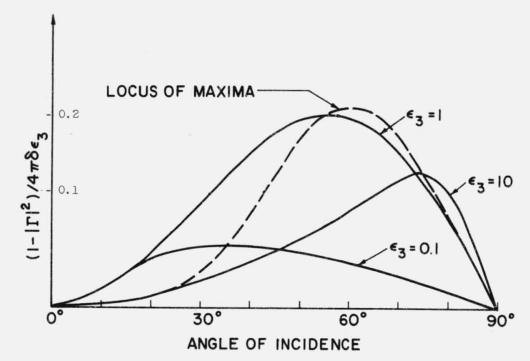

FIGURE 3. Absorption in a thin transition layer.

Applying this boundary condition permits us to evaluate $\gamma$ in the normalized general solution (6)

$$
\gamma \approx \frac{\sqrt{\epsilon_{3} k_{0}^{2}+k_{x}^{2}}}{2 \epsilon_{3} \delta^{1 / 3} k_{0}}\left[1-\frac{\sqrt{\epsilon_{3} k_{0}^{2}+k_{x}^{2}} \epsilon_{3}}{2 k_{0}} \delta+0\left(\delta^{2}\right)\right]
$$

where only the linear term in $\delta$ was retained in the bracket since the present discussion applies to the thin, rapid transition only. By matching boundary conditions at $x_{1}$, we obtain the reflection coefficient in region $\mathrm{I}, \Gamma$, again to first order in $\delta$

$$
\begin{gathered}
\Gamma=\frac{-\epsilon_{3} \sqrt{k_{0}^{2}-k_{x}^{2}}+j \sqrt{\epsilon_{3} k_{0}^{2}+k_{x}^{2}}}{\epsilon_{3} \sqrt{k_{0}^{2}-k_{x}^{2}}+j \sqrt{\epsilon_{3} k_{0}^{2}+k_{x}^{2}}}\left[1-\delta \frac{2 \epsilon_{3} \sqrt{\left(k_{0}^{2}-k_{x}^{2}\right)\left(\epsilon_{3} k_{0}^{2}+k_{x}^{2}\right)}(Q+j P)}{\epsilon_{3}^{2}\left(k_{0}^{2}-k_{x}^{2}\right)+\epsilon_{3} k_{0}^{2}+k_{x}^{2}}+0\left(\delta^{2}\right)\right] \\
Q=\frac{\pi \epsilon_{3} k_{x}^{2} / k_{0}^{2}}{\sqrt{\epsilon_{3}+k_{x}^{2} / k_{0}^{2}}} \\
P=\frac{2}{3 \pi} Q \ln \delta-\frac{Q}{2 \pi}-\frac{\epsilon_{3} \sqrt{\epsilon_{3}+k_{x}^{2} / k_{0}^{2}}}{2}\left(1+\epsilon_{3}^{2}\right) .
\end{gathered}
$$

The power absorption coefficient is the quantity of physical interest here; we obtain

$$
1-|\Gamma|^{2} \underset{\epsilon_{3}^{2}}{\approx} \frac{\pi \delta\left(k_{x}^{2} / k_{0}^{2}\right) \epsilon_{3}^{2} \sqrt{1-k_{x}^{2} / k_{0}^{2}}}{\left(1-k_{x}^{2} / k_{0}^{2}\right)+\epsilon_{3}+k_{x}^{2} / k_{0}^{2}} .
$$

One can obtain this result most conveniently by considering the energy transport associated with the wave propagated in the inhomogeneous medium. The dependence of this fractional power loss (divided by the factor $4 \pi \delta \epsilon_{3}$ ), on angle of incidence is shown on figure 3 . Since $\delta \epsilon_{3}$ remains constant if the transition layer thickness remains constant, these curves represent the dependence of loss on angle of incidence and plasma density for a fixed transition layer thickness. We see that maximum absorption occurs at an angle of incidence of about $60^{\circ}$ and $\epsilon_{3} \approx 1.7$.

\section{Connection Across the Singular Point}

To obtain connection formulas for asymptotic forms of the solution of (5) we resort to Langer's method [Langer, 1949]. In order to eliminate the first derivative term, we transform the dependent variable

$$
U(x)=x^{-1 / 2} H(x)
$$

so that

$$
d^{2} U / d x^{2}-\left[x+B+3 /\left(4 x^{2}\right)\right] U=0 .
$$


The comparison equation must have identical singular and constant terms and an appropriate monotonic term in the coefficient of $U$. The equation which will be used here has a quadratic rather than linear term. This has the consequence that, in order to accomplish the change of sign of that term at the origin, we must employ different comparison equations for $x>0$ and $x<0$. The procedure to be followed is thus going to be slightly more complicated than the usual phase integral method. In the first step, we derive the approximate behavior of the solution at $x=0$ from the boundary condition at $x \longrightarrow+\infty$. Normally we would, say, evaluate the logarithmic derivative (or impedance) at $x=0$; in the present case this is zero for all solutions of the differential equation. As was mentioned above the information needed in our case is the value of the coefficient $\gamma$. Once the value of $\gamma$ is obtained, we make use of it as a boundary condition at the origin to obtain the asymptotic solution for $x \longrightarrow-\infty$.

In the region $0<x<\infty$, we will obtain an approximation to $U$ in terms of the solution of the equation

$$
d^{2} V_{+} / d \xi^{2}-\left[\alpha \xi^{2}+B+3 /\left(4 \xi^{2}\right)\right] V_{+}=0 .
$$

The relationship between $\xi$ and $x$ is the usual one in phase integral procedures:

$$
\int_{0}^{x} \sqrt{x^{\prime}+B+3 / 4\left(x^{\prime}\right)^{2}} d x^{\prime}=\int_{0}^{\xi} \sqrt{\alpha\left(\xi^{\prime}\right)^{2}+B+3 /\left[4\left(\xi^{\prime}\right)^{2}\right]} d \xi^{\prime}
$$

which, for small $x$, reduces to

$$
\xi(x) \approx x\left[1+\frac{2}{9} x^{3}+0\left(x^{4}\right)\right]
$$

The solution of (15) which decays exponentially as $\xi \longrightarrow \infty$ is related to the Whittaker function [Buchholz, 1953] $W_{\lambda, \mu}$ by

$$
V_{+}=C_{1} \xi^{-1 / 2} W_{\frac{-B}{4 \sqrt{\alpha}, \frac{1}{2}}}\left(\sqrt{\alpha} \xi^{2}\right)
$$

One can verify readily that (16) and (17) define a function which has the same asymptotic character for large positive $x$ as $U$, has the same behavior at the origin, and satisfies a differential equation which differs in the coefficient of the term in $U$ by a function which is everywhere small compared to unity [Langer, 1949]. The parameter $\alpha$ has been left undetermined, so far. Let us compare the series expansions for the exact solution of (5) and the approximation in terms of Whittaker functions. We have

$$
H(x)=H(0)\left[1+\frac{B}{2} x^{2} \ln x+\gamma x^{2}+0\left(x^{3}\right)\right]
$$

and

$$
h^{+}(x)=\frac{C_{1}}{\Gamma(1+B / 4 \sqrt{\alpha})}\left[1+\frac{B}{2} x^{2} \ln x+\gamma_{+}(B) x^{2}+0\left(x^{3}\right)\right]
$$

where

$$
\gamma_{+}(B)=\frac{B}{4}\left[\psi(1 B / 4 \sqrt{\alpha})+2 C_{e}-\frac{5}{2}+\frac{1}{2} \ln \alpha\right]-\frac{\sqrt{\alpha}}{2},
$$

$\Gamma$ is the Gamma function, $\psi$ its logarithmic derivative, and $C_{e}$ Eulers constant. 


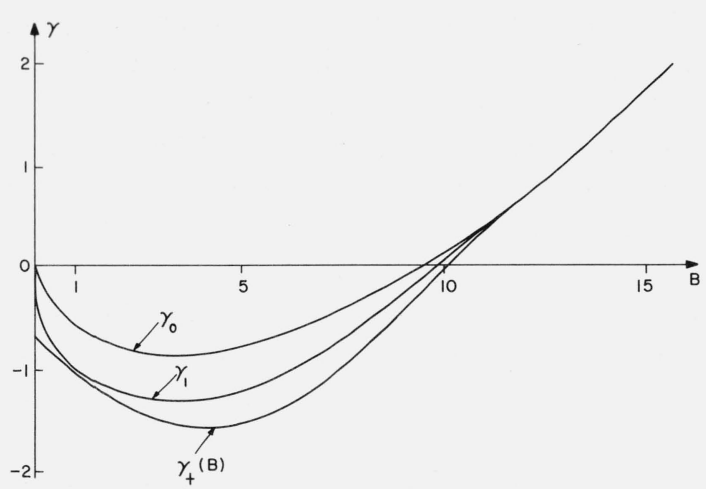

FIGURE 4. Three approximations to $\gamma(\mathrm{B})$.

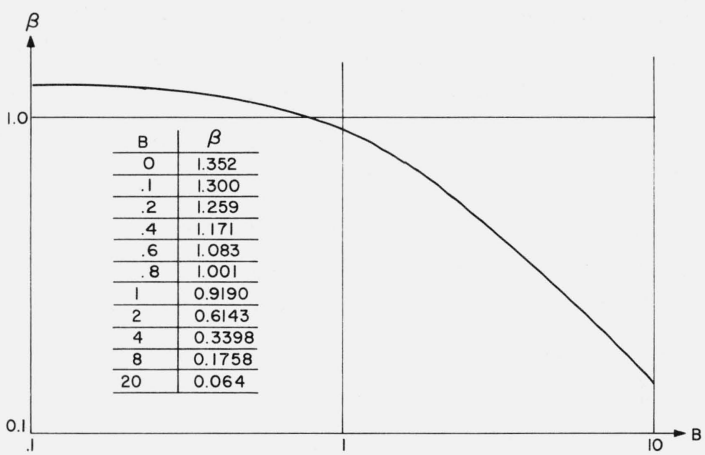

Figure 5. Plot of $\beta(\mathrm{B})$.

The constant $\gamma$ in the exact solution is a function of $B$. If we regard $\alpha$ in (20) as a constant and evaluate it so that $\gamma_{+}(0)$ is exact then it turns out that $\gamma_{+}(B)$ is a good approximation to $\gamma(B)$ for all values of $B$. We obtain $\gamma_{+}(0)$ (in terms of Airy functions) from the known solution of the normal incidence problem [Budden, 1961]. Figure 4 shows a comparison between the function $\gamma_{+}(B)$ thus obtained and another approximation in terms of Hankel functions used by Denisov [1957]. The zero order approximation is $\gamma_{0}$; the first order approximation, obtained by converting the differential equation into an integral equation and iterating once, is $\gamma_{1}$. We see that for large values of $B, \gamma_{o}, \gamma_{1}$, and $\gamma_{+}$are nearly identical. The agreement between $\gamma_{1}$ and $\gamma_{+}$is good for all $B$ except at $B=0$, where $\gamma_{1}$ is known to be in error and $\gamma_{+}$is exact.

Having calculated $\gamma$, we can now determine the asymptotic form of the solution for $x \rightarrow-\infty$. In this case the comparison equation is

$$
d^{2} V_{-} / d \eta^{2}-\left(-\beta \eta^{2}+B+\frac{3}{4 \eta^{2}}\right) V_{-}=0
$$

The transformation of variables is again obtained by equating the phase integrals

$$
\int_{0}^{y} \sqrt{-y^{\prime}+B+3 /\left[4\left(y^{\prime}\right)^{2}\right]} d y^{\prime}=\int_{0}^{\eta} \sqrt{-\beta\left(\eta^{\prime}\right)^{2}+B+3 /\left[4\left(\eta^{\prime}\right)^{2}\right]} d \eta^{\prime}
$$

where

$$
y=|x| \text {. }
$$

Here the constant $\beta$ is determined, and not arbitrary as in the preceding case, by the requirement that (22) must map the turning point of (14) into the turning point of (21). Thus, if $y_{0}$ and $\eta_{0}$ are the zeros of the radicands in (22), $\beta$ is determined by

$$
\int_{0}^{y_{0}} \sqrt{-y+B+3 /\left(4 y^{2}\right)} d y=\int_{0}^{\eta_{0}} \sqrt{-\beta \eta^{2}+B+3 /\left(4 \eta^{2}\right)} d \eta \text {. }
$$

Thus $\beta$ is a function of $B$. The solution of (23) is shown in figure 5 .

The function $U(x)$ is now approximated for $x<0$ by

$$
V_{-}(\eta)=C_{2} \eta^{-1 / 2} W_{\frac{-j B}{4 \sqrt{\beta}} \frac{1}{2}}\left(-j \sqrt{ } \beta \eta^{2}\right)+C_{3} \eta^{-1 / 2} W_{\frac{j B}{4 \sqrt{\beta}}, \frac{1}{2}}\left(j \sqrt{ } \beta \eta^{2}\right)
$$

where $\eta$ is given by (22). The magnitude of the reflection coefficient is simply the magnitude of the ratio $C_{3} / C_{2}$. This is determined by expanding the approximation (24) in a power series, nor- 
Figure 6. Power reflection coefficient from a linearly stratified plasma.

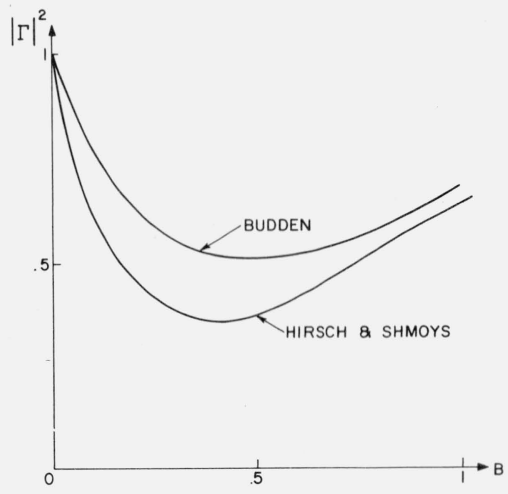

malizing it to unity at $x=0$, and equating the coefficient of the $x^{2}$ term to $\gamma_{+}(B)$. The result is

$$
|\Gamma|=\left|\frac{C_{3}}{C_{2}}\right|=\left|\frac{\gamma_{+}+\frac{j \pi B}{2}-\gamma_{-}}{\gamma_{+}+\frac{j \pi B}{2}-\gamma_{-}^{*}}\right|
$$

where $\gamma$ is given by (20) and $\gamma_{-}$is defined as follows:

$$
\gamma_{-}(B)=\frac{B}{4}\left[\psi\left(1+\frac{j B}{4 \sqrt{\beta}}\right)+2 C_{e}-\frac{5}{2}-j \frac{\pi}{2}+\frac{1}{2} \ln \beta\right]+\frac{j \sqrt{\beta}}{2} .
$$

The magnitude of the reflection coefficient thus calculated is shown in figure 6 , in comparison with results obtained by Budden [1963] ${ }^{2}$ by numerical integration of the differential equation. The agreement between the two curves is good.

${ }^{2}$ Private communication (unpublished numerical data).

We are indebted to L. B. Felsen for valuable critique and stimulating discussion. The research which forms the basis of this paper was sponsored by the Air Force Office of Scientific Research of the Office of Aerospace Research, under Contract No. AF-49(638)-1402.

\section{References}

Buchholz, H. (1953), Konfluente Hypergeometrische Funktion (Springer, Verlag, Berlin).

Budden, K. G. (1961), Radio waves in the ionosphere (Cambridge University Press, Cambridge, U.K.).

Denisov, N. G. (1957), On a singularity of the field of an electromagnetic wave propagated in an inhomogeneous plasma, Soviet Physics JETP 4, 544.

Försterling, K. (1949), Die Ausbreitung elektromagnetischer Wellen in einem geschichteten Medium unter der Mitwerkung eines Magnetfeldes bei schiefer Inzidenz, Archiv der. Elektr. Uber. 3, 115.

Ginzburg, V. L. (1961), Propagation of electromagnetic waves in plasma (Gordon and Breach, New York, N.Y.).

Langer, R. E. (1949), The asymptotic solutions of ordinary linear differential equations of the second order, with special reference to a turning point, Trans. Am. Math. Soc. 67, 461-490.

Wait, J. R. and Walters, L. C. (1963), Reflection of VLF radio waves from an inhomogeneous ionosphere, Part I, J. Res. NBS 67D (Radio Prop.) No. 3, 361-367. 